\title{
U.S. Pneumonia and Influenza Mortality Surveillance: A New Era
}

\author{
Krista Kniss*1, Bianca Malcolm², Paul Sutton² and Lynnette Brammer ${ }^{1}$ \\ ${ }^{1}$ Centers for Disease Control and Prevention, Influenza Division, Atlanta, GA, USA; ${ }^{2}$ Centers for Disease Control and Prevention, \\ National Center for Health Statistics, Division of Vital Statistics, Hyattsville, MD, USA
}

\section{Objective}

To increase the accuracy, completeness, and detail of data as well as decrease the resources needed to conduct pneumonia and influenza mortality surveillance in the U.S.

\section{Introduction}

The 122 Cities Mortality Reporting System (CMRS) has been used for pneumonia and influenza monitoring in the U.S. since the early 20th century. The 122 CMRS is regarded as the timeliest source of mortality data, with the majority of deaths being reported to the system within two weeks. However, while it excels at timeliness it lacks detail, accuracy and completeness. Deaths are counted during the week that the death certificate was filed and not during the week in which the death occurred and the system only covers approximately $25 \%$ of the U.S. population. Also, while the standard case definition for 122 CMRS is a death in which pneumonia or influenza is listed anywhere on the death certificate; not all sites follow this definition (i.e. some sites only use pneumonia or influenza listed only as the underlying cause of death) [1]. The Centers for Disease Control and Prevention's National Center for Health Statistics (NCHS) collects data from state vital statistics offices regarding all deaths that occur in the United States. These data are considered the gold standard of mortality data, but historically had a two year lag due to reporting delays and extensive cleaning and coding of the data [2]. With the advent of electronic death registration (EDR) systems, deaths can now be reported to NCHS in a much timelier manner. Currently NCHS and its partners are funding a number of state projects intended to increase the use of EDR systems. States accepting this funding commit to making significant improvements in the timeliness with which they submit mortality data to NCHS. The goal of this and other activities is to insure all states have the capacity to submit fact of death to NCHS within 3-5 days of death and cause of death within 5-10 days. This combined with significant improvements in NCHS internal IT systems will allow NCHS to provide mortality surveillance data to the Influenza Division on a near real-time basis. We wanted to compare the NCHS mortality surveillance data to the 122 CMRS data and also look at the timeliness of the NCHS data.

\section{Methods}

For the NCHS data, a pneumonia or influenza death is a death with any mention of an ICD-10 multiple-cause code for pneumonia or influenza. Because the NCHS data are collected at a county level, we first took a subset of the NCHS data based on the county equivalents for each of the 122 cities that report data to CDC. We then compared the percent P\&I from both streams starting October 2008 through December 2012 both visually as well as calculated a Pearson correlation coefficient. We also looked at the median number of days between death and fact of death transmission to NCHS for 2005-2009 to evaluate past and current timeliness of the NCHS data.

\section{Results}

Comparing the percent of deaths per week due to pneumonia and influenza for the NCHS and 122 CMRS data, there is good correlation
(0.76), but the two data streams do not match exactly. The percent of deaths due to pneumonia and influenza was always higher in the NCHS data than in the 122 CMRS data. Looking at the timeliness of the data, the time between death and when NCHS received fact of death was a median of 91 days ( 13 weeks) with a range of 0-955 days. During the timeframe (2005-2009) this lag greatly improved from a median of 117 days in 2005 to a median of 76 days in 2009.

\section{Conclusions}

Assuming that the timeliness of the NCHS data continues to increase at the same rate as it did during the 2005-2009 time period as well as the relative correlation of the data, we think that the NCHS data stream could potentially be a valid replacement for the 122 CMRS. The NCHS data would monitor deaths by the date of death, would cover $100 \%$ of all deaths occurring in the U.S., and all deaths would be coded and counted in the same way. We plan to monitor both systems throughout the next influenza season to check for the accuracy and feasibility of using the NCHS mortality data as a surveillance tool. Future analyses will also assess the reliability of the NCHS real-time surveillance data by comparing weekly P\&I ratios based on incomplete surveillance data to the ratios computed from final annual NCHS data.

\section{Keywords}

Electronic Death Registration System; Mortality Surveillance; Pneumonia and Influenza; National Center for Health Statistics

\section{References}

[1] 122 Cities Mortality Reporting System: http://www.cdc.gov/flu/ weekly/overview.htm\#Mortality

[2] NCHS Background: http://www.cdc.gov/nchs/deaths.htm

\author{
*Krista Kniss \\ E-mail: krk9@cdc.gov
}

